\title{
Thrombotic Microangiopathy and Venous Thrombosis in a Patient With Anti-Neutrophil Cytoplasmic Antibody-Associated Vasculitis
}

\author{
Khanh Duong ${ }^{1}$, Samantha Etienne ${ }^{1}$, Roberto Collazo-Maldonado ${ }^{2}$, Irina Lytvak ${ }^{3}$ \\ 1. Department of Internal Medicine, Methodist Dallas Medical Center, Dallas, USA 2. Department of Nephrology, \\ Methodist Dallas Medical Center, Dallas, USA 3. Department of Pathology, Methodist Dallas Medical Center, Dallas, \\ USA
}

Corresponding author: Khanh Duong, khanhduong88@gmail.com

\begin{abstract}
Antineutrophil cytoplasmic antibody (ANCA)-associated vasculitis (AAV) is a systemic pauci-immune small vessel vasculitis. Its various presentations make AAV diagnosis challenging. Here, we present a case of AAV with thrombotic microangiopathy (TMA) and deep venous thrombosis (DVT). An 82-year-old Hispanic woman presented to the emergency department with malaise, lower extremity pain, and oliguria for three days. Her vital signs were normal, and her physical examination was unremarkable. The initial laboratory revealed thrombocytopenia $\left(18 \times 10^{3} / \mu \mathrm{L}\right)$, elevated creatinine $(8.35 \mathrm{mg} / \mathrm{dL})$, high lactic acid dehydrogenase $(1627.5 \mathrm{U} / \mathrm{L})$, an international normalized ratio of 1.6 , and an activated partial thromboplastin time of 49 seconds. Urinalysis showed microscopic hematuria and proteinuria, and peripheral smear revealed schistocytes. She was admitted with concern for TMA. Further workup revealed an antinuclear antibody titer of 1:80, an ADAMTS13 level of 62\%, a rheumatoid factor level of $151.7 \mathrm{IU} / \mathrm{L}$, and myeloperoxidase (MPO)ANCA level of $173 \mathrm{AU} / \mathrm{mL}$. A computed tomography scan of the chest/abdomen/pelvis revealed pulmonary fibrosis and multifocal consolidations. She was also found to have extensive DVT of the lower extremities. Renal biopsy revealed early changes of TMA with one cellular crescent. She was diagnosed with AAV based on the kidney and lung findings, as well as the high titer MPO-ANCA. Her platelet count and creatinine improved significantly following treatment with plasma exchange, steroids, and rituximab. Unfortunately, she was then found to have an acute bowel perforation and expired. Even though typically rare, an increased incidence of venous thromboembolism (VTE) and TMA has been reported in patients with AAV. Its prompt recognition and treatment by clinicians are critical to mitigate the unfavorable outcomes from this condition.
\end{abstract}

Review began 11/04/2020 Review ended 11/12/2020 Published 11/23/2020

\section{() Copyright 2020}

Duong et al. This is an open access article distributed under the terms of the Creative Commons Attribution License CC-BY 4.0., which permits unrestricted use, distribution, and reproduction in any medium, provided the original author and source are credited.
Categories: Internal Medicine, Nephrology, Rheumatology

Keywords: vasculitis, thrombotic microangiopathy, venous thrombosis, anti-neutrophil cytoplasmic antibodyassociated vasculitis, anca

\section{Introduction}

Antineutrophilic cytoplasmic antibody (ANCA)-associated vasculitis (AAV) is a systemic vasculitis with unknown etiology. AAV is a pauci-immune small vessel vasculitis and its various presentations can make diagnosis challenging. An increased incidence of venous thromboembolism (VTE) and thrombotic microangiopathy (TMA) associated with AAV have been reported. Here, we present a case of AAV with TMA and deep venous thrombosis (DVT).

\section{Case Presentation}

An 82-year-old Hispanic woman with a past medical history of hypertension presented to the emergency department with malaise for three days. She also experienced nausea, vomiting, fatigue, lower extremity pain, shortness of breath, cough, and oliguria. No diarrhea, weight loss, history of malignancy, or kidney disease were reported. Her vital signs were normal, and her physical examination was unremarkable. The results of initial labs revealed low platelets with concomitant elevated creatinine, lactic acid dehydrogenase, international normalized ratio, and activated partial thromboplastin time; her haptoglobin level was undetectable (Table 1). A peripheral smear showed red blood cell schistocytes consistent with microangiopathic hemolytic anemia. Urinalysis showed microscopic hematuria and proteinuria. She was admitted with the diagnosis of microangiopathic hemolytic anemia and acute kidney injury. A non-tunneled dialysis catheter was placed and she underwent plasma exchange and was given steroids. Stool collection for Escherichia coli 0157:H7 testing was not feasible as this patient did not have a bowel movement during hospital admission. She was later found to have an extensive lower extremity DVT, and heparin was administered. A workup for malignancy was ordered along with computed tomography (CT) scan of the chest/abdomen/pelvis. The CT scan revealed a pulmonary fibrosis pattern and multifocal areas of consolidation in the upper lung (Figure 1). Tumor markers (CEA, CA 19-9, CA 125, AFP) were negative. Rituximab was started as the patient's ANCA results were positive. Her platelet count and creatinine levels improved significantly following treatment (Table 2). 


\section{Cureus}

\begin{tabular}{|c|c|c|}
\hline Test name & Reference range & Lab value \\
\hline \multicolumn{3}{|l|}{ Hematology } \\
\hline $\mathrm{Hb}(\mathrm{g} / \mathrm{dL})$ & $12.0-16.0$ & 14.5 \\
\hline Platelet count $\left(\times 10^{3} / \mu \mathrm{L}\right)$ & $130-400$ & 18 \\
\hline Haptoglobin (mg/dL) & $30-200$ & $<20$ \\
\hline LDH (U/L) & $313-618$ & 1627.5 \\
\hline D-Dimer, Quant $(\mu \mathrm{g} / \mathrm{mL})$ & $0.00-0.50$ & $>20.00$ \\
\hline Fibrinogen (mg/dL) & $214-481$ & 92 \\
\hline aPTT (seconds) & $23-37$ & 49 \\
\hline PT & $11.3-14.7$ & 19.1 \\
\hline INR & $0.9-1.2$ & 1.6 \\
\hline \multicolumn{3}{|l|}{ Rheumatology panel } \\
\hline ANA titer & Negative at 1:40 & $1: 80$ \\
\hline ENA antibodies screen (EU) & $\leq 20.000$ & 4.123 \\
\hline Anti-DNA antibody (IU) & $\leq 25.000$ & 14.220 \\
\hline Rheumatoid factor (IU/mL) & $\leq 12.0$ & 151.7 \\
\hline Cryoglobulin quant, blood & Negative & Negative \\
\hline GBM Ab IgG (AU/mL) & $0-19$ & 0 \\
\hline Sm antibody (AU/mL) & $0-40$ & 5 \\
\hline Anti-Scl70 IgG (AU/mL) & $0-40$ & 0 \\
\hline Myositis antibody & Negative & Negative \\
\hline ANCA IgG & $<1: 20$ & 1:1280 \\
\hline MPO-ANCA (AU/mL) & $0-19$ & 173 \\
\hline PR3-ANCA (AU/mL) & $0-19$ & 1 \\
\hline Direct antiglobulin test & & Negative \\
\hline ADAMTS13 & $50-160 \%$ & $62 \%$ \\
\hline \multicolumn{3}{|l|}{ Inflammatory markers } \\
\hline Procalcitonin (ng/mL) & $<0.25$ & 0.28 \\
\hline CRP (mg/L) & $\leq 10$ & 31 \\
\hline ESR (mm) & $0-20$ & 1 \\
\hline C3 (mg/dL) & $88-165$ & 121 \\
\hline C4 (mg/dL) & $14-44$ & 27 \\
\hline \multicolumn{3}{|l|}{ Infectious workup } \\
\hline HIV Ab & & Negative \\
\hline HCV Ab & & Negative \\
\hline HbsAg & & Negative \\
\hline Blood culture & & No growth at five days \\
\hline \multicolumn{3}{|l|}{ Tumor markers } \\
\hline CEA (ng/mL) & $\leq 3.0$ & 1.0 \\
\hline
\end{tabular}




\section{Cureus}

CA $19-9(\mathrm{U} / \mathrm{mL})$

CA 125 (U/mL)

$\operatorname{AFP}(n g / m L)$

Others

Vitamin B12 (pg/mL)

Folate (ng/mL) $\leq 37.00$

18.20

$\leq 35$

12

$<7.51$

2.11

$211-911$

908

$3.0-20.0$

17.6

\section{TABLE 1: The patient's initial workup}

ADAMTS13: a disintegrin and metalloproteinase with a thrombospondin type 1 motif, member 13; AFP: alpha-fetoprotein; ANA: antinuclear antibody; ANCA: antineutrophil cytoplasmic antibodies; aPTT: activated partial thromboplastin time; CA: cancer antigen; CEA: carcinoembryonic antigen; CRP: C-reactive protein; ENA: extractable nuclear antigen; ESR: erythrocyte sedimentation rate; GBM: glomerular basement membrane; HBsAg: hepatitis B surface antigen; Hb: hemoglobin; HCV: hepatitis C virus; HIV: human immunodeficiency virus; LDH: lactate dehydrogenase; MPO: myeloperoxidase antibodies; PT: prothrombin time.

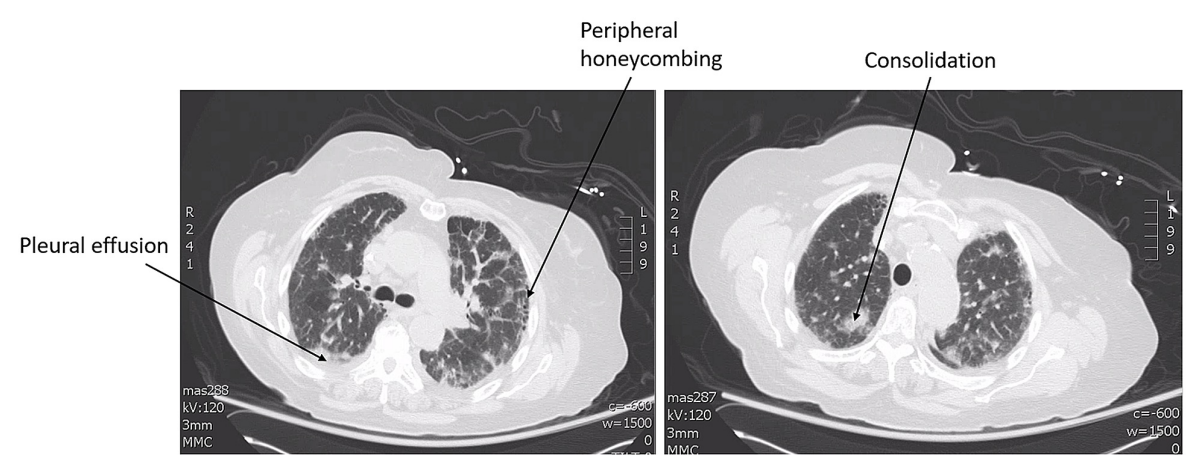

FIGURE 1: Computed tomography scan of the chest/abdomen/pelvis showed pulmonary fibrosis pattern, small bilateral perfusion, and multifocal areas of consolidation

\section{Test name}

Creatinine (mg/dL)

Platelet $\left(\times 10^{3} / \mu \mathrm{L}\right)$

\section{Reference range}

$0.7-1.3$

$130-400$

\begin{tabular}{|l|}
\hline Day 1 \\
\hline 8.35
\end{tabular}

18

Day 2
6.92
9

Day 3
4.90
42

Day 4

Day 5

Day 6 Day 7

3.46

127

2.20

1.80

$\begin{array}{lll}108 & 127 & 112\end{array}$

226

\section{TABLE 2: Kidney-function-and-platelet-level-monitoring-during-the-patient's-length-of-stay}

A kidney biopsy was done following the resolution of her thrombocytopenia. Light microscopic examination of the kidney specimen revealed 23 glomeruli, of which six were globally sclerotic, one demonstrated early glomerular capillary thrombosis, and another demonstrated a cellular crescent (Figure 2). There was no evidence of vasculitis, but moderate arteriosclerosis was present. Mild interstitial fibrosis and tubular atrophy were found in the tubulointerstitium, involving $10 \%$ to $15 \%$ of the renal cortical tissue. Electron microscopy showed no electron-dense immune-type deposits or organized deposits. Immunofluorescent staining (IgG, IgA, IgM, C3, C1q, kappa, and lambda) of the glomeruli were negative. 


\section{Cureus}

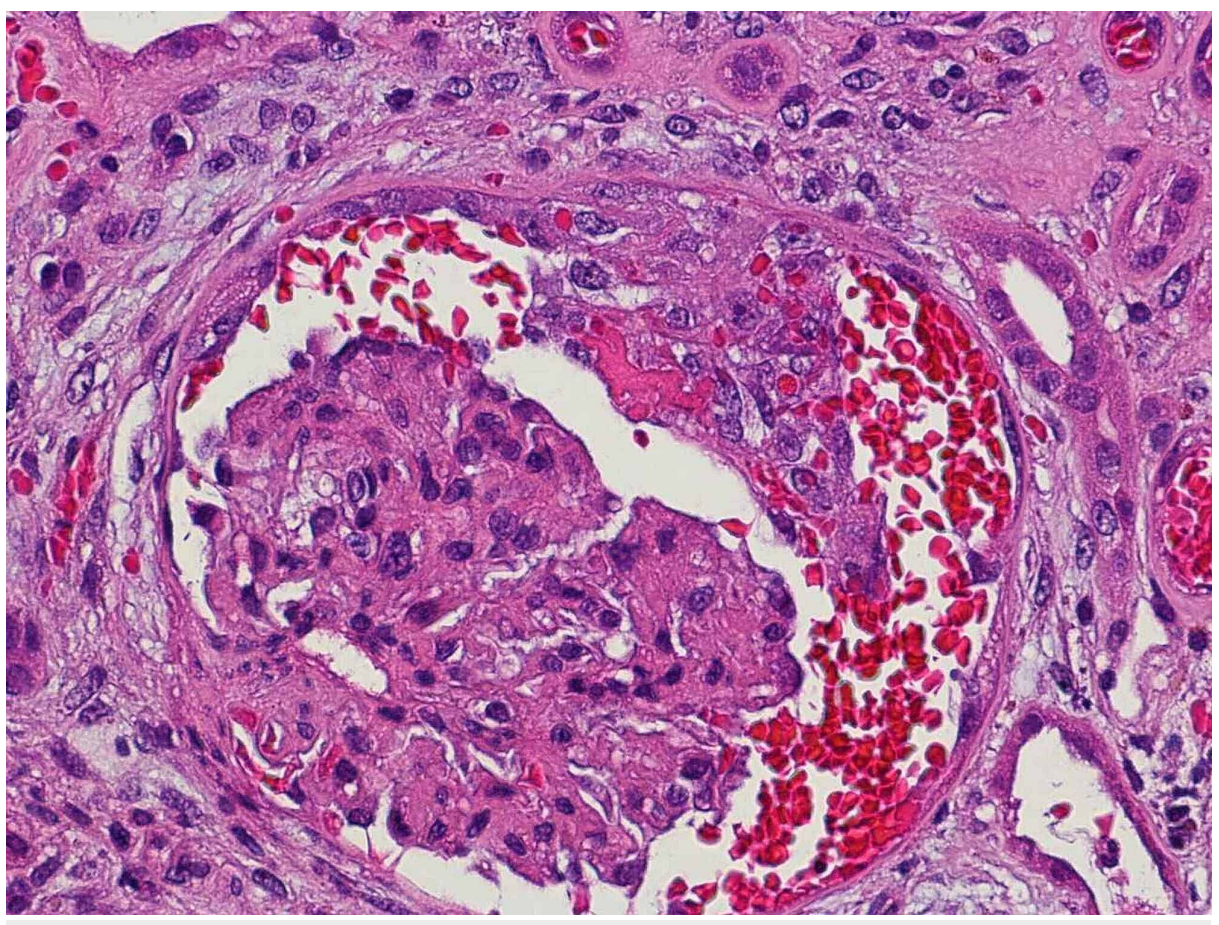

\section{FIGURE 2: Kidney biopsy showed glomerulus with a cellular crescent (H\&E stain)}

During the course of treatment, this patient developed an acute bowel perforation not amenable to treatment and expired.

\section{Discussion}

Our patient was admitted with TMA and DVT. At first, malignancy was an obvious option for the differential diagnoses; however, the workup was negative. An infection workup was also negative. This patient was diagnosed with AAV based on the kidney and lung findings as well as the very high titer of MPO-ANCA, consistent with microscopic polyangiitis (MPA). Pulmonary involvement is observed in $25 \%$ of patients with MPA, including diffuse alveolar hemorrhage, interstitial lung disease (ILD), and pleuritis [1,2]. ILD is present in about $7.2 \%$ of patients at the time of MPA diagnosis [1]. Approximately $80 \%$ of patients with MPA have renal manifestations [2]. Global glomerulosclerosis (defined as sclerotic changes in the glomerulus that compose $>80 \%$ of the tuft) is common and can be seen in around 30\% of MPA patients [3]. Glomerular crescents are present in approximately $65 \%$ of patients [3]. Findings in the kidney biopsy that support the AAV diagnosis in this patient included focal crescentic glomerulonephritis and global glomerulosclerosis using light microscopy and pauci-immune staining pattern using immunofluorescence. Vasculitis and fibrinoid necrosis are often seen in less than $20 \%$ of patients with MPA [3]; these were not seen in the biopsy specimen of this patient's kidney.

TMA is characterized by abnormalities in the vessel wall of arterioles and capillaries that lead to microvascular thrombosis [4]. Primary TMA syndromes include thrombotic thrombocytopenic purpura, Shiga toxin-mediated hemolytic uremic syndrome (HUS), drug-mediated TMA, complement-mediated TMA (alternatively, atypical HUS), coagulation-mediated TMA, and metabolism-mediated TMA [5]. Secondary TMA can be seen in systemic infections, systemic cancer, severe preeclampsia, eclampsia, hemolysis, elevated liver enzymes, low platelet syndrome, severe hypertension, autoimmune disorders (e.g., systemic lupus erythematosus, systemic sclerosis, antiphospholipid syndrome), and hematopoietic stem-cell or organ transplantation [5]. Our patient had evidence of TMA on lab tests and kidney biopsy. Furthermore, lab tests/history revealed a normal ADAMTS13 level, normal vitamin B12 level, lack of diarrhea/infection, and no contributing medication use. The severe kidney involvement with evidence of TMA on the kidney biopsy made complement-mediated TMA a possible diagnosis. Her response to plasmapheresis and steroids also pointed to this diagnosis. Unfortunately, we failed to demonstrate complement dysregulation.

AAV-associated TMA has been described in several case reports [6-10]. The etiology is unknown, but the endovascular damage due to vasculitis and complement pathway abnormalities could be the triggers. AAV patients with concomitant renal TMA presented with more severe renal injury. Also, TMA was independently associated with all-cause mortality in patients with AAV [11]. Recent studies demonstrated an increased risk of thromboembolic events in AAV. The risk of VTE is over three times greater in patients with AAV than in the general population [12]. The risk of arterial thrombotic disease is also increased. The 
risk of cardiovascular disease is over three times greater and the risk for a cerebrovascular accident is eight times greater in patients with AAV than in the general population [12]. These risks are higher during the active phase, supporting the role of inflammation during the thrombosis in promoting a prothrombotic state [13].

Our patient responded very well to treatment; her creatinine and platelet levels normalized. Unfortunately, she developed acute bowel perforation. Bowel perforation has been reported to occur in about $15 \%$ of systemic small and medium-sized vessel vasculitides with gastrointestinal tract involvement [14].

\section{Conclusions}

Even though typically rare, an increased incidence of VTE and TMA has been reported in patients with AAV. To the best of our knowledge, this is the first report of a patient with AAV that developed both TMA and DVT concomitantly in the active phase. Prompt recognition and intensive treatment with steroids, plasmapheresis, and rituximab treatment are critical to mitigate unfavorable outcomes.

\section{Additional Information}

\section{Disclosures}

Human subjects: All authors have confirmed that this study did not involve human participants or tissue. Conflicts of interest: In compliance with the ICMJE uniform disclosure form, all authors declare the following: Payment/services info: All authors have declared that no financial support was received from any organization for the submitted work. Financial relationships: All authors have declared that they have no financial relationships at present or within the previous three years with any organizations that might have an interest in the submitted work. Other relationships: All authors have declared that there are no other relationships or activities that could appear to have influenced the submitted work.

\section{References}

1. Arulkumaran N, Periselneris N, Gaskin G, Strickland N, Ind PW, Pusey CD, Salama AD: Interstitial lung disease and ANCA-associated vasculitis: a retrospective observational cohort study. Rheumatology (Oxford). 2011, 50:2035-43. 10.1093/rheumatology/ker236

2. Guillevin L, Durand-Gasselin B, Cevallos R, et al.: Microscopic polyangiitis: clinical and laboratory findings in eighty-five patients. Arthritis Rheum. 1999, 42:421-30. 10.1002/1529-0131(199904)42:3<421::AIDANR5 $>3.0 . \mathrm{CO} ; 2-6$

3. Hauer HA, Bajema I, Houwelingen H, et al.: Renal histology in ANCA-associated vasculitis: differences between diagnostic and serologic subgroups. Kidney Int. 2002, 61:80-9. 10.1046/j.1523-1755.2002.00089.x

4. Laszik ZG, Kambham N, Silva FG, et al.: Thrombotic microangiopathies. Heptinstall's Pathology of the Kidney. Jennett JC, D'Agati VD, Olson JL, et al. (ed): Lippincott Williams \& Wilkins, Philadelphia; 2014

5. George JN, Nester CM: Syndromes of thrombotic microangiopathy. N Engl J Med. 2014, 371:654-66. 10.1056/NEJMra1312353

6. Agrawal V, Vaidya CK, Ye J, et al.: Concomitant thrombotic thrombocytopenic purpura and ANCAassociated vasculitis in an adolescent. Pediatr Nephrol. 2011, 26:1317-20. 10.1007/s00467-011-1862-5

7. Lim HE, Jo SK, Kim SW, et al.: A case of Wegener's granulomatosis complicated by diffuse pulmonary hemorrhage and thrombotic thrombocytopenic purpura. Korean J Intern Med. 1998, 13:68-71. 10.3904/kjim.1998.13.1.68

8. Nagai K, Kotani T, Takeuchi T, et al.: Successful treatment of thrombotic thrombocytopenic purpura with repeated plasma exchange in a patient with microscopic polyangiitis. Mod Rheumatol. 2008, 18:643-6. 10.1007/s10165-008-0107-3

9. Stefanidis I, Helmchen U, Schmitt H, Maurin N, Sieberth HG: Coincidence of haemolytic uraemic syndrome and c-ANCA-associated rapidly progressive glomerulonephritis. Nephrol Dial Transplant. 1998, 13:1818-21. 10.1093/ndt/13.7.1818

10. Yamauchi Y, Nagatoya K, Okuno A, Fujii N, Inoue T: Successful treatment for thrombotic thrombocytopenic purpura complicated with myeloperoxidase anti-neutrophil cytoplasmic autoantibody-associated vasculitis. NDT Plus. 2010, 3:279-81. 10.1093/ndtplus/sfq013

11. Chen SF, Wang H, Huang YM, et al.: Clinicopathologic characteristics and outcomes of renal thrombotic microangiopathy in anti-neutrophil cytoplasmic autoantibody-associated glomerulonephritis. Clin J Am Soc Nephrol. 2015, 10:750-8. 10.2215/CJN.07910814

12. Berti A, Matteson EL, Crowson CS, Specks U, Cornec D: Risk of cardiovascular disease and venous thromboembolism among patients with incident ANCA-associated vasculitis: a 20-year population-based cohort study. Mayo Clin Proc. 2018, 93:597-606. 10.1016/j.mayocp.2018.02.010

13. Springer J, Villa-Forte A: Thrombosis in vasculitis. Curr Opin Rheumatol. 2013, 25:19-25. 10.1097/BOR.0b013e32835ad3ca

14. Pagnoux C, Mahr A, Cohen P, Guillevin L: Presentation and outcome of gastrointestinal involvement in systemic necrotizing vasculitides: analysis of 62 patients with polyarteritis nodosa, microscopic polyangiitis, Wegener granulomatosis, Churg-Strauss syndrome, or rheumatoid arthritis-associated vasculitis. Medicine. 2005, 84:115-28. 10.1097/01.md.0000158825.87055.0b 